\title{
Is South Africa at risk for Zika virus disease?
}

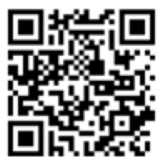

Zika virus (ZIKV) was originally isolated from a sentinel rhesus monkey in the Zika forest in Uganda in $1947 . .^{[1]}$ Twenty-one years later the virus was isolated for the first time from naturally infected humans in Nigeria. ${ }^{[2]}$ ZIKV belongs to the genus Flavivirus, family Flaviviridae, comprising enveloped viruses with RNA genomes, mostly arthropod borne. ${ }^{[3]}$

For decades ZIKV remained relatively unknown, affecting mainly monkeys and occasionally causing a mild disease in humans residing across a narrow equatorial belt in Africa and Asia. ${ }^{[4]}$ The virus is transmitted to humans by daytime active Aedes aegypti mosquitoes and usually causes a self-limiting, non-fatal febrile infection characterised by a maculopapular rash, arthralgia, conjunctivitis, myalgia and headache. ${ }^{[5]}$

From 2007 the geographical distribution of ZIKV expanded drastically, with first outbreaks documented in some of the Pacific islands, including the Federated States of Micronesia. Additional Pacific islands were affected in 2013 - 2014, followed by unprecedented spread of the virus in the Americas from 2014. ${ }^{[4,6]}$ As of February 2016, 23 countries in Latin America and the Caribbean had reported active transmission of ZIKV. In Brazil alone, estimation of ZIKV infections in 2015 ranged from 500000 to 1.5 million. ${ }^{[7]}$ This situation is concerning in view of the explosive spread of ZIKV outside its traditional geographical boundaries, and is further complicated by supposed association of ZIKV infections with birth malformations and neurological syndromes. Drastic increases in the birth of babies with abnormally small heads (microcephaly) and unilateral ophthalmological abnormalities, and cases of GuillainBarré syndrome, are reported in some areas recently affected by the virus. ${ }^{[8]}$ The newly postulated risks of ZIKV infection, with a potential high burden on families and communities, are of great international concern. On 1 February 2016 the WHO proclaimed the rise in cases of microcephaly and neurological disorders reported in Brazil as a public health emergency of international concern. This declaration calls for a more co-ordinated effort to improve surveillance, improve mosquito control programmes and fast-track the development of diagnostic assays, vaccines and antivirals for ZIKV and to scientifically investigate the causality of the observed disorders. ${ }^{[9]}$

The clinical presentation of ZIKV disease is not characteristic enough to enable even a tentative diagnosis. A known history of recent travel to an area with active ZIKV transmission is informative, and helps to guide specific referral laboratory testing. There is no specific prophylaxis other than prevention of mosquito bites, no specific antivirals are available, and treatment is supportive. Infection rarely leads to hospitalisation, and it is believed that up to $80 \%$ of infected individuals remain asymptomatic. While investigations to determine the underlying pathology of ZIKV infection are ongoing, pregnant women are discouraged from travelling to affected areas at any stage of pregnancy. Two cases of possible sexual transmission of ZIKV have been reported. The most recent is associated with the first case of ZIKV disease diagnosed in a traveller (returning from Venezuela) and diagnosis of ZIKV disease in his sexual partner who did not travel outside the USA (unofficial report at time of publication). It would be reasonable to consider precautions (i.e. abstinence, condom use) for pregnant women with sexual partners who have travelled to affected areas and could be affected or are confirmed with ZIKV infection. ${ }^{[10]}$ Two cases of probable perinatal transmission are also on record from French Polynesia (2013). ${ }^{[11]}$ The potential for ZIKV transmission through blood transfusion has been demonstrated from the outbreak in French Polynesia (2013). ${ }^{[12]}$

The risk of ZIKV transmission in any given area partly depends on the availability of competent mosquito vectors. A. aegypti mosquitoes are the major transmitters of $\mathrm{ZIKV}$, as for dengue viruses. While causing an increased number of outbreaks in Africa and Asia in recent years, none of the known dengue virus types is endemic in South Africa (SA). Considering that the risk of transmission of the two related viruses is likely to be the same, the prediction models based on the distribution of $A$. aegypti therefore do not place SA at high risk for transmission of ZIKV. It should be emphasised here that different populations of $A$. aegypti mosquitoes may have dissimilar competence in vectoring these viruses to humans. No human cases of infection with ZIKV related to local transmission have been diagnosed in SA to date. The first imported case of ZIKV disease in SA was confirmed in the week of 15 February 2016, in a traveller from Colombia with mild illness. Further imported cases in travellers visiting affected areas can be expected. Dengue cases have only been diagnosed in travellers returning home from countries with active circulation of the virus. ${ }^{[13]} \mathrm{A}$. aegypti mosquitoes are found in SA, particularly in the eastern coastal plain but also in the cities of the inland plateau. In urban areas these mosquitoes breed in small collections of water such as in discarded tyres and buckets, or the leaf axils of banana trees. There is a possibility that a traveller infected with ZIKV may return to SA. The transient presence of virus in the blood (viraemia), however, decreases the likelihood of human-to-mosquito transmission and the establishment of an autochthonous transmission cycle.

Patients presenting with the symptoms described above and with a history of recent ( $<2$ weeks) travel to an affected area should consider ZIKV disease, but also related infections such as chikungunya or dengue, as a possible diagnosis. Infection with these viruses can be confirmed by specialised referral laboratory testing at the National Institute for Communicable Diseases (NICD). Malaria should also be considered. Laboratory testing for confirmation of ZIKV infection at the NICD requires the submission of serum or clotted blood along with a comprehensive clinical and travel history. Blood/serum collected up to day 5 after the onset of disease is most suitable for confirmation of acute infection by virus culture and detection of ZIKV virus nucleic acid by reverse transcription-polymerase chain reaction (RT-PCR) assay. Although RT-PCR is highly sensitive and specific, its application in laboratory confirmation of ZIKV infection is limited by the short duration of viraemia ( $2-4$ days). Serological diagnosis of ZIKV infection is complicated by the high level of cross-reaction with other flaviviruses. For this reason laboratory confirmation of infection based on serological results is only feasible through the testing of paired serum samples taken at least 14 days apart, to demonstrate a four-fold rise in antibody titre. Parallel serological testing for dengue virus antibodies is also important and diagnostic interpretation hinges on the quantitative comparison of these results. Rapid diagnostic tests (point-of-care assays) are not currently available.

In response to the increasing spread of ZIKV and the potential high health threats it poses, the following measures need to be considered: $(i)$ detection and monitoring of virus dissemination 
based on event-based surveillance; (ii) vector management; (iii) preparedness of healthcare settings and services for the management of complicated ZIKV cases; (iv) risk communication and public awareness; $(v)$ training and capacity building for clinical and laboratory diagnosis, vector control and communication; and (vi) research and development to address increasing needs for diagnostic reagents and rapid diagnostic kits.

\section{Petrus Jansen van Vuren, Jacqueline Weyer, Alan Kemp,}

Veerle Dermaux-Msimang

Centre for Emerging and Zoonotic Diseases, National Institute for Communicable Diseases, National Health Laboratory Service, Sandringham, Johannesburg, South Africa

\section{Kerrigan McCarthy, Lucille Blumberg}

Division of Public Health Surveillance and Response, National Institute for Communicable Diseases, National Health Laboratory Service, Sandringham, Johannesburg, South Africa

\section{Janusz Paweska}

Centre for Emerging and Zoonotic Diseases, National Institute for Communicable Diseases, National Health Laboratory Service, Sandringham, Johannesburg, South Africa
Acknowledgement. Allison Glass, Lancet Laboratories, Johannesburg.

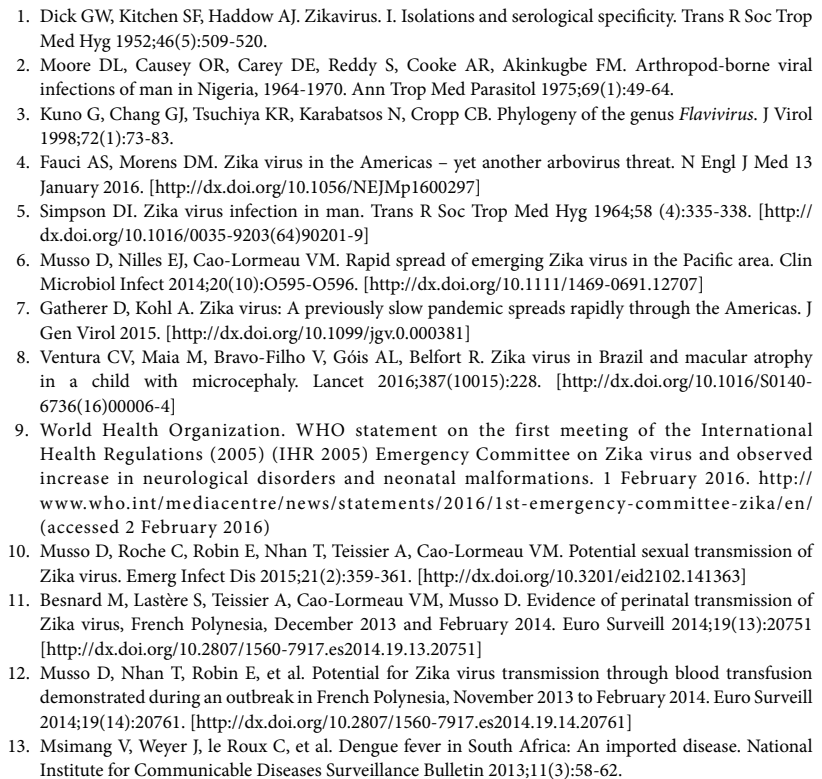

This month in the $S A M J \ldots$

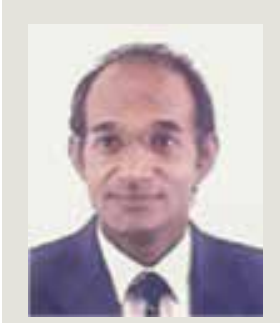

Datshana Prakash Naidoo* is Chief Specialist in and Head of the Department of Cardiology at the Nelson R Mandela School of Medicine, University of KwaZulu-Natal, and Inkosi Albert Luthuli Hospital, Durban. He is Past President of the Southern African Hypertension Society, holds a doctorate in cardiology, and is a Fellow of the Royal College of Physicians. A National Research Foundation-rated researcher, his research interests are directed towards establishing the role of genetic and environmental factors in the pathogenesis of coronary artery disease. He has pioneered the training of cardiac technologists in echocardiography and is responsible for establishing a non-invasive cardiology service across KZN.

*Prakaschandra DR, Esterhuizen TM, Motala AA, Gathiram P, Naidoo DP. High prevalence of cardiovascular risk factors in Durban South African Indians: The Phoenix Lifestyle Project. S Afr Med J 2016;106(3):284-289. [http://dx.doi.org/10.7196/SAMJ.2016.v106i3.9837]

Andrew Redfern* is a developmental paediatrician currently working in the Paediatric Emergency and Ambulatory Department at Tygerberg Children's Hospital, Cape Town. He received his MPhil in developmental paediatrics from the University of Cape Town in 2014. His areas of interest at the time were improving the provision of services for children with disability. Currently he finds himself on a new path, with growing interests in the fields of paediatric emergency medicine and medical education. He is passionate about free open-access medical education, simulation and improving paediatric emergency care and training for all children in South Africa and beyond.

*Redfern A, Westwood A, Donald KA. Children with disabling chronic conditions in the Western health subdistrict of Cape Town, South Africa: Estimating numbers and service gaps. S Afr Med J 2016;106(3):302-307. [http://dx.doi.org/10.7196/SAMJ.2016.v106i3.9825]

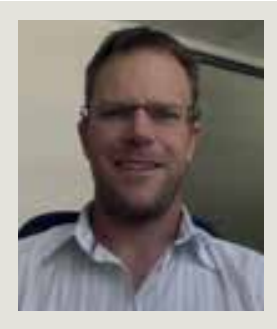

